The Astrophysical Journal, 512:L51-L54, 1999 February 10

(C) 1999. The American Astronomical Society. All rights reserved. Printed in U.S.A.

\title{
DETECTION OF PRESHOCK DENSE CIRCUMSTELLAR MATERIAL OF SN 1978K
}

\author{
You-Hua Chu ${ }^{1}$ and Adeline Caulet \\ Astronomy Department, University of Illinois, 1002 West Green Street, Urbana, IL 61801; chu@astro.uiuc.edu, caulet@astro.uiuc.edu \\ Marcos J. Montes \\ Naval Research Laboratory, Code 7212, Washington DC 20375-5320; montes@ rsd.nrl.navy.mil \\ Nino Panagia \\ Space Telescope Science Institute, 3700 San Martin Drive, Baltimore, MD 21218; panagia@stsci.edu \\ SCHUYLER D. VAN DYK \\ Infrared Processing and Analysis Center/Caltech, Mail Code 100-22, Pasadena, CA 91125; vandyk@ipac.caltech.edu \\ AND \\ KURT W. WEILER \\ Naval Research Laboratory, Code 7214, Washington DC 20375-5320; weiler@rsd.nrl.navy.mil \\ Received 1998 October 19; accepted 1998 December 11; published 1999 January 13
}

\begin{abstract}
The supernova SN 1978K has been noted for its lack of emission lines broader than a few thousand kilometers per second since its discovery in 1990. Modeling of the radio spectrum of the peculiar SN 1978K indicates the existence of $\mathrm{H}$ II absorption along the line of sight. To determine the nature of this absorbing region, we have obtained a high-dispersion spectrum of SN $1978 \mathrm{~K}$ at the wavelength range $6530-6610 \AA$. The spectrum shows not only the moderately broad $\mathrm{H} \alpha$ emission of the supernova ejecta but also narrow nebular $\mathrm{H} \alpha$ and [N II] emission. The high $\left[\mathrm{N}_{\mathrm{II}}\right] \lambda 6583 / \mathrm{H} \alpha$ ratio, $0.8-1.3$, suggests that this radio-absorbing region is a stellar ejecta nebula. The expansion velocity and emission measure of the nebula are consistent with those seen in ejecta nebulae of luminous blue variables. Previous low-dispersion spectra have detected a strong [N II] $\lambda 5755$ line, indicating an electron density of (3-12) $\times 10^{5} \mathrm{~cm}^{-3}$. We argue that this stellar ejecta nebula is probably part of the preshock dense circumstellar envelope of SN 1978K. We further suggest that SN 1997ab may represent a young version of SN 1978K.
\end{abstract}

Subject headings: circumstellar matter — galaxies: individual (NGC 1313) — stars: mass loss supernovae: individual (SN 1978K)

\section{INTRODUCTION}

Massive stars lose mass via stellar winds throughout their lifetime. Stellar winds expand away from the stars and form circumstellar envelopes. As a massive star ends its life in a supernova ( $\mathrm{SN}$ ) explosion, the $\mathrm{SN}$ ejecta plows through the circumstellar material, driving a forward shock into the circumstellar material and a reverse shock into the SN ejecta. Optical emission is generated in the ionized SN ejecta, in cooled SN ejecta behind the reverse shock, in shocked circumstellar material, and in the ambient ionized circumstellar material (Chevalier \& Fransson 1994). These four regions have different physical conditions and velocity structures. Consequently, optical luminosities and spectral characteristics of Type II SNe not only vary rapidly for individual SNe, but also differ widely among SNe with different progenitors.

Optical spectra of Type II SNe older than a few years are characterized by broad hydrogen Balmer lines and oxygen forbidden lines, with FWHMs greater than a few thousand kilometers per second, reflecting the rapid expansion of the SN ejecta (e.g., SN 1979C and SN 1980K: Fesen et al. 1999; SN 1986E: Cappellaro, Danziger, \& Turatto 1995; SN 1987F: Filippenko 1989; SN 1994aj: Benetti et al. 1998). Some Type II SNe, however, do not seem to show such broad emission lines. The most notable case is SN 1978K.

SN 1978 K in NGC 1313 was discovered in 1990 during a

\footnotetext{
${ }^{1}$ Visiting astronomer, Cerro Tololo Inter-American Observatory, National Optical Astronomy Observatories, operated by the Association of Universities for Research in Astronomy, Inc., under a cooperative agreement with the National Science Foundation.
}

spectrophotometric survey of extragalactic H II regions (Ryder \& Dopita 1993). Ryder et al. (1993) examined archival optical images of NGC 1313 and established that the optical maximum of the supernova occurred in 1978, possibly two months before July 31. However, the optical spectra of SN $1978 \mathrm{~K}$ obtained in 1990-1992 do not show any emission line broader than 600 $\mathrm{km} \mathrm{s}^{-1}$ (Ryder et al. 1993; Chugai, Danziger, \& Della Valle 1995). This is in sharp contrast to SN 1980K, which shows broad, $6000 \mathrm{~km} \mathrm{~s}^{-1}$ emission lines in spectra obtained in 1988 and 1997 (Fesen et al. 1999).

SN $1978 \mathrm{~K}$ is intriguing at radio wavelengths as well. While its radio flux shows temporal variations consistent with the expectation of a typical Type II SN, its radio spectrum shows a low-frequency turnover that is most plausibly caused by freefree absorption from an $\mathrm{H}$ II region along the line of sight (Ryder et al. 1993). Montes, Weiler, \& Panagia (1997) reanalyzed the radio observations of SN $1978 \mathrm{~K}$ and find that the intervening $\mathrm{H}$ II region has an emission measure $\mathrm{EM}=$ $8.5 \times 10^{5}\left(T_{e} / 10^{4} \mathrm{~K}\right)^{1.35} \mathrm{~cm}^{-6} \mathrm{pc}$, where $T_{e}$ is the electron temperature.

To determine the nature of this "H II region" toward SN 1978K, we have obtained a high-dispersion echelle spectrum at the wavelength range of $6530-6610 \AA$. This spectrum clearly resolves the narrow [N II] $\lambda \lambda 6548,6583$ lines and a narrow $\mathrm{H} \alpha$ component from a moderately broad $\mathrm{H} \alpha$ component. The narrow $\mathrm{H} \alpha$ and [N $\mathrm{NI}$ ] lines must arise from the $\mathrm{H}$ II region, and the broad $\mathrm{H} \alpha$ component must arise from the SN ejecta. In this Letter, we report the echelle observation $(\S$ $2)$, compare our spectrum with previous low-dispersion spectra (§ 3), argue that the H II region toward SN 1978K is circum- 
stellar, and suggest a feasible explanation for SN 1978K's apparent lack of very broad emission lines (§ 4$)$.

\section{HIGH-DISPERSION SPECTRUM OF SN 1978K}

We obtained a high-dispersion spectrum of SN 1978K using the echelle spectrograph on the $4 \mathrm{~m}$ telescope at Cerro Tololo Inter-American Observatory (CTIO) on 1997 February 27. The spectrograph was used in a long-slit, single-order mode; the cross disperser was replaced by a flat mirror, and a broad $\mathrm{H} \alpha$ filter $(\mathrm{FWHM}=75 \AA$ ) was inserted behind the slit. The slit width was $250 \mu \mathrm{m}$, or 1".64. The data were recorded with the red long-focus camera and a Tektronix $2048 \times 2048$ CCD. The pixel size was $0.08 \AA$ pixel $^{-1}$ along the dispersion and 0.26 pixel $^{-1}$ in the spatial axis. The instrumental FWHM was $14 \pm 1 \mathrm{~km} \mathrm{~s}^{-1}$. The data were wavelength-calibrated but not flux-calibrated.

The echelle observation of SN $1978 \mathrm{~K}$ was made with a 10 minute exposure. SN 1978K and two unrelated H II regions are detected. No spatially extended $\mathrm{H}$ II features exist at the position of SN 1978K. A spectrum extracted from a $5^{\prime \prime}$ slit length ${ }^{2}$ centered on SN 1978K is presented in Figure 1. The high-dispersion spectrum shows three sets of lines with distinct velocity widths. The narrowest (unresolved) are the telluric $\mathrm{H} \alpha$ and $\mathrm{OH} \lambda 6553.617$ and $\lambda 6577.285$ lines (Osterbrock et al. 1996). The broadest is the $\mathrm{H} \alpha$ emission from the supernova ejecta. It is centered at $6572.76 \pm 0.22 \AA$, corresponding to a heliocentric velocity $\left(V_{\text {hel }}\right)$ of $455 \pm 10 \mathrm{~km} \mathrm{~s}^{-1}$; its FWHM is $\sim 450 \mathrm{~km} \mathrm{~s}^{-1}$ and its FWZI is $\sim 1100 \mathrm{~km} \mathrm{~s}^{-1}$.

The third set of lines consists of the narrow [N II] $\lambda \lambda 6548$, 6583 lines and a narrow $\mathrm{H} \alpha$ component. The narrow $\mathrm{H} \alpha$ component is superposed near the peak of the broad $\mathrm{H} \alpha$ emission of the supernova, hence its central velocity $\left(V_{\text {hel }} \sim 419 \mathrm{~km} \mathrm{~s}^{-1}\right)$ and FWHM (75-100 $\mathrm{km} \mathrm{s}^{-1}$ ) are somewhat uncertain. The [N II] $\lambda 6583$ line, at $V_{\text {hel }}=419 \pm 5 \mathrm{~km} \mathrm{~s}^{-1}$, shows a line split of $\sim 70 \mathrm{~km} \mathrm{~s}^{-1}$; its FWHM is $\sim 125 \mathrm{~km} \mathrm{~s}^{-1}$. The [N II] $\lambda 6548$ line, being weaker, does not show an obvious line split; however, its asymmetric line profile indicates the presence of a brighter red component and a weaker blue component, consistent with those seen in the [N II] $\lambda 6583$ line.

The narrow $\mathrm{H} \alpha$ component and the narrow [N II] lines most likely originate from the same emitting region and will be referred to as "nebular" emission. We have measured the nebular [N II] $\lambda 6583 / \mathrm{H} \alpha$ ratio to be $0.8-1.3$. The large uncertainty in this ratio is caused by the uncertainty in the nebular $\mathrm{H} \alpha$ flux, since it is difficult to separate the nebular and supernova contributions to the observed $\mathrm{H} \alpha$ emission. The possible range of nebular $[\mathrm{N} \mathrm{II}] / \mathrm{H} \alpha$ ratio is derived from the lower and upper limits of the nebular $\mathrm{H} \alpha$ flux, estimated by assuming high and low peaks of supernova emission, respectively.

\section{COMPARISON WITH PREVIOUS LOW-DISPERSION SPECTRA}

A relatively low-dispersion spectrum of SN 1978K was obtained on 1990 January 23 by Ryder et al. (1993). That spectrum showed an $\mathrm{H} \alpha$ line centered at $6570.2 \pm 0.6 \AA$ with an FWHM of $563 \mathrm{~km} \mathrm{~s}^{-1}$. It also detected the [N II] $\lambda 6583$ line at $6589.6 \pm 1.0 \AA$. Since this spectrum has a resolution of $\sim 5$ $\AA$ and a pixel size of $1.5 \AA$ pixel $^{-1}$, the [N II] lines are not well resolved from the $\mathrm{H} \alpha$ line and consequently the velocity and flux measurements might not be very accurate. The [N II]

\footnotetext{
${ }^{2}$ This large slit length is necessary to include all the light from SN 1978K, since the telescope was slightly out of focus for this observation. When the focus problem was resolved ( $2 \mathrm{hr}$ later), SN 1978K had already set.
}

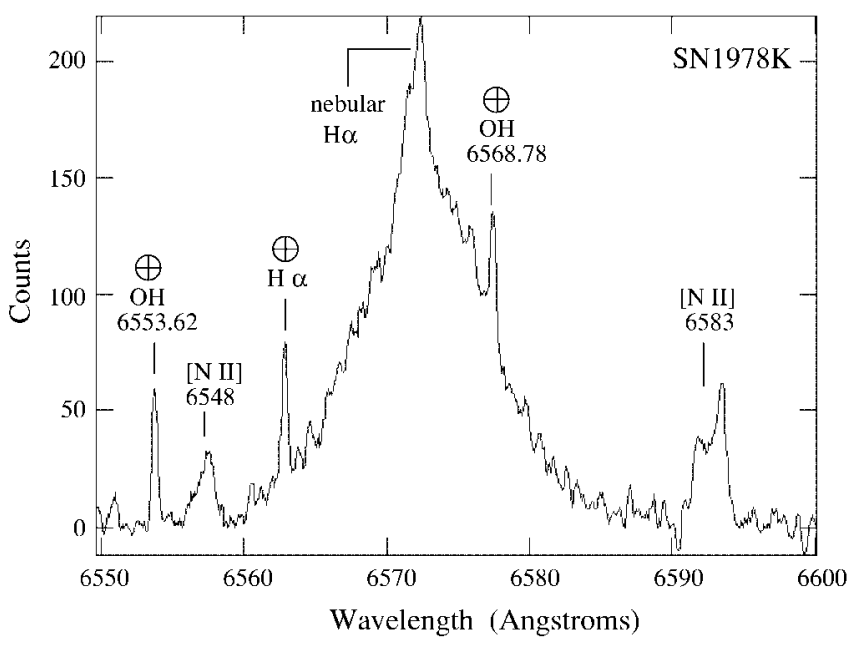

FIG. 1.-High-dispersion spectrum of SN 1978K taken with the echelle spectrograph on the CTIO $4 \mathrm{~m}$ telescope. The spectrum has been smoothed with a boxcar of 5 pixels, or $0.4 \AA$. The telluric lines are indicated by the circled plus signs.

$\lambda 6583 / \mathrm{H} \alpha$ flux ratio, 0.049 , derived from this low-dispersion spectrum is really the ratio of nebular $[\mathrm{N} \mathrm{II]} \lambda 6583$ flux to the combined supernova and nebular $\mathrm{H} \alpha$ flux. The [N II] $\lambda 5755$ line is also detected and the $[\mathrm{N} \mathrm{II}] \lambda 5755 / \mathrm{H} \alpha$ flux ratio is 0.025 .

Another low-dispersion spectrum of SN 1978K was obtained on 1992 October 22 by Chugai et al. (1995). The resolution of this spectrum is $10 \AA$. Thus, the redshifts and widths of spectral lines cannot be reliably determined. The $[\mathrm{N}$ II] $\lambda 6583$ / $\mathrm{H} \alpha$ flux ratio is 0.072 , and the [N II] $\lambda 5755 / \mathrm{H} \alpha$ flux ratio is 0.016 .

Using our echelle spectrum, we have measured the ratio of nebular [N II] $\lambda 6583$ flux to the combined supernova and nebular $\mathrm{H} \alpha$ flux to be 0.06 . This is different from the previous measurements, 0.049 and 0.072. While our measurement should be more accurate because of our higher spectral resolution, the supernova $\mathrm{H} \alpha$ flux might have varied from 1990 to 1997 (Chugai et al. 1995). It is not clear whether the [N II] flux itself has changed.

Nebular lines toward SN 1978K are also detected in the UV spectra of SN 1978K obtained with the Faint Object Spectrograph on board the Hubble Space Telescope on 1994 September 26 and 1996 September 22-23 (Schlegel et al. 1999). The Ly $\alpha$ line and the blended [Ne IV] $\lambda \lambda 2421,2424$ doublet are detected. Both lines have FWHMs comparable to the instrumental resolution (7 $\AA$ ), corresponding to $1727 \mathrm{~km} \mathrm{~s}^{-1}$ at Ly $\alpha$ and 866 $\mathrm{km} \mathrm{s}^{-1}$ at [Ne IV]. These [Ne IV] lines have critical densities of $8 \times 10^{4}$ and $2.5 \times 10^{5} \mathrm{~cm}^{-3}$, respectively (Zheng 1988); therefore, these [Ne IV] lines must originate from the nebula. The Ly $\alpha$ line emission, like the $\mathrm{H} \alpha$ emission, contains both the supernova ejecta and nebular components.

\section{DISCUSSION}

\subsection{Origin of the Narrow $\mathrm{H} \alpha$ and [N II] Lines}

The most intriguing features detected in our high-dispersion spectrum of SN 1978K are the narrow nebular $\mathrm{H} \alpha$ and [N II] lines, which are presumably emitted by the "H II region along the line of sight" implied by the radio spectrum of SN 1978K (Ryder et al. 1993). However, as we argue below, the [N II] line strengths suggest that this $\mathrm{H}$ II region is circumstellar, rather than interstellar. 
The nebular $[\mathrm{N}$ II $] \lambda 6583 / \mathrm{H} \alpha$ line ratio $(0.8-1.3)$ is unusually high for normal interstellar $\mathrm{H}$ II regions in a spiral galaxy. For example, $\mathrm{H}$ II regions in M101 have [N II] $\lambda 6583 / \mathrm{H} \alpha$ ratios $\leq 0.3$ (Kennicutt \& Garnett 1996). SN $1978 \mathrm{~K}$ is at the outskirts of NGC 1313, where abundances are expected to be low and the $\mathrm{H}$ II excitation is expected to be high. If the nebular $\mathrm{H} \alpha$ and [N II] lines toward SN 1978K originate in an interstellar $\mathrm{H}$ II region, we would expect the $[\mathrm{N} \mathrm{II}] \lambda 6583 / \mathrm{H} \alpha$ ratio to be $\sim 0.1$ or lower. A low interstellar $[\mathrm{N} \mathrm{II}] / \mathrm{H} \alpha$ ratio is confirmed by the bright $\mathrm{H}$ II region detected along the slit at $\sim 90^{\prime \prime}$ east of SN 1978K. This H II region is brighter than the nebula toward SN $1978 \mathrm{~K}$ in the $\mathrm{H} \alpha$ line, but its [N II] $\lambda 6583$ line is not detected. We may rule out an interstellar $\mathrm{H}$ II region explanation for the narrow nebular lines seen in SN 1978K.

The high $[\mathrm{N}$ II] $\lambda 6583 / \mathrm{H} \alpha$ ratio may be caused by a high electron temperature or a high nitrogen abundance. These conditions can be easily provided by SN $1978 \mathrm{~K}$ and its progenitor. If the nebula was ionized by the UV flash of SN 1978K, the electron temperature may be higher than that of a normal $\mathrm{H}$ II region, as in the case of SN 1987A's outer rings (Panagia et al. 1996). However, the [N II] $\lambda 6583$ line intensity increases by only a factor of 2 for an electron temperature increase from 10,000 to $15,000 \mathrm{~K}$. This increase cannot explain fully the observed high $[\mathrm{N}$ II $] / \mathrm{H} \alpha$ ratio. A higher nitrogen abundance is needed. An elevated nitrogen abundance is characteristic of ejecta nebulae around evolved massive stars, such as luminous blue variables (LBVs) and Wolf-Rayet (W-R) stars; the [N II] $\lambda 6583 / \mathrm{H} \alpha$ ratios of these ejecta nebulae are frequently observed to be $\sim 1$ (Esteban et al. 1992; Smith et al. 1998). Therefore, the most reasonable origin of the nebular emission lines toward SN $1978 K$ would be a circumstellar ejecta nebula. The observed high $[\mathrm{N} \mathrm{II}] / \mathrm{H} \alpha$ ratio may be caused by the combination a high nitrogen abundance and a high electron temperature.

SN 1978K's circumstellar ejecta nebula has a very high density, since a strong [N $\mathrm{NI}] \lambda 5755$ line is observed in SN 1978K's spectrum. The [N II] $(\lambda 6548+\lambda 6583) / \lambda 5755$ ratio is measured to be 2.55 by Ryder et al. (1993) and 6.0 by Chugai et al. (1995), indicating that collisional deexcitation is significant for the ${ }^{1} D_{2}$ level of $\mathrm{N}^{+}$. If we assume an electron temperature of $(1-1.5) \times 10^{4} \mathrm{~K}$, the observed [N II] line ratios imply electron densities of $(3-12) \times 10^{5} \mathrm{~cm}^{-3}$. The circumstellar ejecta nebula of SN $1978 \mathrm{~K}$ can be compared to those observed around LBVs and W-R stars. The density of SN 1978K's nebula is higher than those of W-R nebulae, but within the range for LBV nebulae (Stahl 1989; Esteban et al. 1992). We adopt the emission measure $\mathrm{EM}=8.5 \times$ $10^{5}\left(T_{e} / 10^{4} \mathrm{~K}\right)^{1.35} \mathrm{~cm}^{-6}$ pc determined from the radio observations (Montes et al. 1997) for SN 1978K's nebula. This emission measure is much higher than those observed in ejecta nebulae around W-R stars, typically a few times $10^{2}-10^{3} \mathrm{~cm}^{-6}$ pc (Esteban et al. 1992; Esteban \& Vílchez 1992), but lies toward the high end of the range typically seen in LBV nebulae, a few times $10^{3}-10^{5} \mathrm{~cm}^{-6}$ pc (Hutsemékers 1994; Smith et al. 1998). Finally, the $\mathrm{H} \alpha$ and [N II] velocity profiles seen in our SN 1978 K spectrum suggest an expansion velocity ${ }^{3}$ of $40-55$ $\mathrm{km} \mathrm{s}^{-1}$, which is lower than those of most ejecta nebulae around W-R stars but is within the range for LBV nebulae (Nota et al. 1995; Chu, Weis, \& Garnett 1999). It is thus likely that the observed nebula toward SN $1978 \mathrm{~K}$ was ejected by the progenitor during a LBV phase before the SN explosion.

\footnotetext{
${ }^{3}$ The expansion velocity implied by the line split in the $[\mathrm{N}$ II] line is greater than $35 \mathrm{~km} \mathrm{~s}^{-1}$. The expansion velocity can also be approximated by the halfwidth at half-maximum of the $\mathrm{H} \alpha$ and $[\mathrm{N} \mathrm{II}]$ lines, $40-55 \mathrm{~km} \mathrm{~s}^{-1}$.
}

This ejecta nebula could be either part of the circumstellar envelope that the SN ejecta expands into, or a shell that is detached from the circumstellar envelope. We will demonstrate below that the latter is unlikely. If the ejecta nebula is a detached shell, the observed emission measure and density imply that the shell thickness is only $4 \times 10^{-5}$ to $4 \times 10^{-6} \mathrm{pc}$. The thickness of a detached, dense shell will be broadened by diffusion and may be crudely approximated by $\left(c / V_{\exp }\right) R$, where $c$ is the isothermal sound velocity, $V_{\exp }$ is the expansion velocity, and $R$ is the radius. We find that the radius of SN 1978K's freeexpanding ejecta shell would have to be no greater than $\sim 2 \times 10^{-4} \mathrm{pc}$, which is smaller than the expected radius of the $\mathrm{SN}$ ejecta. This is impossible. Therefore, we conclude that the narrow $\mathrm{H} \alpha$ and $[\mathrm{N}$ II] lines must originate in the preshock, ionized circumstellar envelope of SN 1978K.

The narrow nebular lines from the preshock, ionized circumstellar envelope of SN 1978K are not unique among SNe. The high-dispersion spectrum of SN 1997ab shows narrow P Cygni $\mathrm{H} \alpha$ and narrow [N II] $\lambda 6583$ lines, and the FWZI of the $\mathrm{P}$ Cyg $\mathrm{H} \alpha$ line $\left(180 \mathrm{~km} \mathrm{~s}^{-1}\right)$ is comparable to that of SN $1978 K$ 's H $\alpha$ line (Salamanca et al. 1998). The P Cyg profile of SN 1997ab's narrow $\mathrm{H} \alpha$ line indicates a high density, $\geq 10^{7}$ $\mathrm{cm}^{-3}$. This density exceeds the critical density of the ${ }^{1} D_{2}$ level of $\mathrm{N}^{+}$and causes a weak [N II] $\lambda 6584$ line (see Fig. 1 of Salamanca et al. 1998). If SN 1997ab's circumstellar material is nitrogen-rich like that of SN $1978 \mathrm{~K}$, we predict that its [N II] $\lambda 5755$ line is strong and should be detectable as well. SN 1997ab is very likely a younger version of SN 1978K, and SN 1978K's nebular H $\alpha$ line may have exhibited a P Cyg profile in 1979-1980.

\subsection{SN Evolution in a Very Dense Circumstellar Envelope}

The most notable SN characteristic of SN 1978K is its apparent lack of very broad (a few thousand kilometers per second) emission lines. Adopting canonical expansion velocities and sizes for SN 1978K, Ryder et al. (1993) have derived a mass of greater than $80 M_{\odot}$ for the circumstellar envelope. This mass is too large to reconcile with the current understanding of massive stellar evolution. To lower the circumstellar mass, Chugai et al. (1995) propose that the circumstellar envelope is clumpy.

We consider that the large size $(\sim 0.1 \mathrm{pc})$ adopted by Ryder et al. (1993) is overestimated and inconsistent with the expansion velocity implied by our observed $\mathrm{H} \alpha \mathrm{FWHM}$ of $450 \mathrm{~km}$ $\mathrm{s}^{-1}$. There is no need to assume an unseen, larger expansion velocity. We suggest that the small expansion velocity of SN $1978 \mathrm{~K}$ is caused by the dense circumstellar envelope, which has quickly decelerated the expansion of SN ejecta. If optical spectra had been obtained immediately after the SN explosion in 1978, very broad emission lines would have been detected.

Rapid deceleration of SN ejecta has been observed in two other SNe, SN 1986J and SN 1997ab. SN 1986J has been noted to have spectral properties very similar to those of SN $1978 \mathrm{~K}^{4}$ SN 1986J probably exploded 4 years before its initial discovery in 1986 (Rupen et al. 1987; Chevalier 1987). Its optical spectra obtained soon after the discovery show narrow hydrogen Balmer lines and nitrogen forbidden lines, indicating an expansion velocity less than $600 \mathrm{~km} \mathrm{~s}^{-1}$ (Leibundgut et al. 1991). SN 1997ab is the only other SN for which narrow nebular

\footnotetext{
${ }^{4}$ We have examined a large number of SN spectra reported in the literature. SN 1986J appears to be the only SN besides SN 1978K that shows strong [N II] $\lambda 5755$ line, indicating a very high density and possibly an enhanced nitrogen abundance.
} 
emission lines from the dense circumstellar envelope have been unambiguously resolved and detected. SN 1997ab's light curve peaked in 1996; the FWHM of its $\mathrm{H} \alpha$ line decreased rapidly from $2500 \mathrm{~km} \mathrm{~s}^{-1}$ on 1997 March 2 to $1800 \mathrm{~km} \mathrm{~s}^{-1}$ on 1997 May 30 (Hagen, Engels, \& Reimers 1997; Salamanca et al. 1998).

Clearly, SN 1978K, SN 1986J, and SN 1997ab all possess very dense circumstellar envelopes, and we may expect them to evolve similarly. The expansion of SN 1978K might have slowed down to below $1000 \mathrm{~km} \mathrm{~s}^{-1}$ within the first $\sim 2 \mathrm{yr}$ after the explosion, and the $\mathrm{SN}$ ejecta could not have reached a radius greater than $\sim 0.02 \mathrm{pc}$ in 1990. A factor of 5 reduction in the radius would lower Ryder et al.'s (1993) estimate of mass to a reasonable value, and the hypothesis of a clumpy circumstellar envelope will no longer be necessary.

\subsection{Future Work}

Previous spectrophotometric observations of $\mathrm{SNe}$ were rarely made with spectral resolutions better than $2 \AA$. Our echelle observation of SN 1978K has demonstrated that highdispersion spectroscopy is powerful in resolving preshock, ion- ized circumstellar material. A high-dispersion spectroscopic survey of young $\mathrm{SNe}$ in nearby galaxies may detect more circumstellar envelopes and even detached ejecta ring nebulae, such as the rings around SN 1987A (Burrows et al. 1995). The density and velocity structures of these circumstellar envelopes would shed light on the mass-loss history as well as physical properties of the massive progenitors.

Our spectrum of SN 1978K unfortunately covers only the [ $\mathrm{N}$ II] and $\mathrm{H} \alpha$ lines. In order to measure the density, temperature, and abundances of the circumstellar material, it is necessary to obtain high-dispersion spectra covering a large wavelength range. It is also important to monitor the spectral changes indicative of density changes in the circumstellar envelope. A large change at all wavelengths is expected when the SN ejecta expands past the outer edge of the circumstellar envelope.

We would like to thank the referee for useful suggestions to improve this Letter. Y. H. C. acknowledges the support of NASA Long-Term Space Astrophysics grant NAG5-3246. M. J. M. and K. W. W. wish to thank the Office of Naval Research for the 6.1 funding supporting this research.

\section{REFERENCES}

Benetti, S., Cappellaro, E., Danziger, I. J., Turatto, M., Patat, F., \& Della Valle, M. 1998, MNRAS, 294, 448

Burrows, C. J., et al. 1995, ApJ, 452, 680

Cappellaro, E., Danziger, I. J., \& Turatto, M. 1995, MNRAS, 277, 106

Chevalier, R. A. 1987, Nature, 329, 611

Chevalier, R. A., \& Fransson, C. 1994, ApJ, 420, 268

Chu, Y.-H., Weis, K., \& Garnett, D. R. 1999, AJ, in press

Chugai, N. N., Danziger, I. J., \& Della Valle, M. 1995, MNRAS, 276, 530

Esteban, C., \& Vílchez, J. M. 1992, ApJ, 390, 536

Esteban, C., Vílchez, J. M., Smith, L. J., \& Clegg, R. E. S. 1992, A\&A, 259, 629

Fesen, R. A., et al. 1999, AJ, in press (astro-ph/9810407)

Filippenko, A. V. 1989, AJ, 97, 726

Hagen, H.-J., Engels, D., \& Reimers, D. 1997, A\&A, 324, L29

Hutsemékers, D. 1994, A\&A, 281, L81

Kennicutt, R. C., Jr., \& Garnett, D. R. 1996, ApJ, 456, 504

Leibundgut, B., et al. 1991, ApJ, 372, 544

Montes, M. J., Weiler, K. W., \& Panagia, N. 1997, ApJ, 488, 792
Nota, A., Livio, M., Clampin, M., \& Schulte-Ladbeck, R. 1995, ApJ, 448, 788 Osterbrock, D. E., Fulbright, J. P., Martel, A. R., Keane, M. J., Trager, S. C., \& Basri, G. 1996, PASP, 108, 277

Panagia, N., Scuderi, S., Gilmozi, R., Challis, P. M., Garnavich, P. M., \& Kirshner, R. P. 1996, ApJ, 459, L17

Rupen, M. P., van Gorkom, J. H., Knapp, G. R., Gunn, J. E., \& Schneider, D. P. 1987, AJ, 94, 61

Ryder, S. D., \& Dopita, M. A. 1993, ApJS, 88, 415

Ryder, S., Staveley-Smith, L., Dopita, M., Petre, R., Colbert, E., Malin, D., \& Schlegel, E. 1993, ApJ, 416, 167

Salamanca, I., Cid-Fernandes, R., Tenorio-Tagle, G., Telles, E., Terlevich, R. J., \& Muñoz-Tuñón, C. 1998, MNRAS, 300, L17

Schlegel, E., et al. 1999, in preparation

Smith, L. J., Nota, A., Pasquali, A., Leitherer, C., Clampin, M., \& Crowther, P. A. 1998, ApJ, 503, 278

Stahl, O. 1989, in Physics of Luminous Blue Variables, ed. K. Davidson, A. F. Moffat, \& H. J. Lamers (Dordrecht: Kluwer), 149

Zheng, W. 1988, Astrophys. Lett. Commun., 27, 275 